\title{
2nd special issue on robust analysis of complex data
}

When we embarked to invite submissions for this 2 nd special issue on robust analysis of complex data, we did so with the following call:

Nowadays we are often confronted with large data sets in high dimensions. These new types of data have led to the emergence

and use of complex models such as graphical models, models for complex correlation structures and models for functional data

for instance. These complex structures pose many challenges. Specifically, when many measurements on several variables are

recorded, it becomes more likely that not all of these measurements are recorded with high accuracy. This may result in data of

uneven quality that contains gross errors and other anomalies that need to be taken into account. Therefore, there is a need for

robust procedures that can reliably analyze large data sets containing outliers and other data contamination. This special issue

will focus mainly on computationally efficient, robust procedures to analyze such complex data sets.

This call resulted in 37 submissions, from which five high quality papers have been selected for inclusion in this special issue. We greatly acknowledge the help of the CSDA co-editors to handle these papers and we thank all anonymous referees

for their valuable comments, honest opinions and constructive suggestions.

Dhaene and Zhu (2017) study the robustness properties of median-based estimators for the AR(1) coefficient in different types of additive outliers scenarios for panel data when the number of time-points is growing to infinity while the number of observed panels is fixed. They do so through showing that the influence function is bounded when outliers occur independently over time or are patched additive, i.e. they occur in random sequences of length $\mathrm{k}$.

Bali and Boente (2017) consider robust principal component estimation of several populations of functional data. They adapt methods from one population functional data, instead of estimating the covariance operator for each of the populations

separately, Bali and Boente (2017) propose to use robust projection-pursuit estimators for the common directions under a common principal component model instead. The benefit is that considerably fewer parameters need to be estimated. A particular highlight of the article is that Bali and Boente (2017) extend the definition of a functional common principal component model to the situation in which the covariance operator does not exist or when the underlying distribution is not elliptical, and show how this still ensures Fisher-consistency.

Chiancone et al. (2017) show how to robustify Sliced Inverse Regression (SIR), which has been shown in Cook (2007) to correspond to the maximum likelihood of an inverse regression model with Gaussian errors, by extending its inverse regression formulation to non-Gaussian errors with heavy tailed distributions. In contrast to alternative robust versions of SIR, Chiancone et al. (2017) do not replace the standard SIR estimators by robust versions but achieve robustness through focussing on the intrinsic characterisation of SIR by working with multivariate t-distributions where estimation of parameters can still be achieved through maximum likelihood estimation via the EM algorithm.

He et al. (2017) investigate the estimation of high dimensional copula graphical model by simultaneous testing wether or not in high-dimensional Gaussian graphical models the ith component of a p-variate multivariate normal distributed random

variable is conditionally independent of the jth component, given all remaining variables. The false discovery rate is used to measure performance and robustness is gained by exploiting nonparametric rank-based tests. The procedure requires that p regression equations are solved, which is done with the Lasso or Dantzig selector Candes and Tao (2007). Maruotti and Punzo (2017) use flexible hidden Markov regression models to analyze heterogeneous longitudinal multivariate responses which may depend on several fixed covariates. To provide robustness against outlying responses, the

standard multivariate Gaussian distribution for the outcome vectors can be replaced by either a multivariate $t$-distribution or a multivariate contaminated Gaussian distribution. This is one of the first contributions providing a robust method to analyze these complex data.

As is the nature of publishing, what is eventually selected is to a high degree independent of competing submissions, yet it is striking that the five selected papers by 'chance' cover the entire range of what was sketched in the original call and more-it is encouraging to see robustness at play in a very different sense.

Since the first special issue on robust analysis of complex data was published in CSDA (Croux et al., 2013) four years ago,

the journal continued to attract a considerable body of work on different aspects of robustness in complex data analysis. These include Alfons et al. (2016), Cui et al. (2016), Hämäläinen (2016), Kirschstein et al. (2016), Martinez and Gray (2016),

Salibián-Barrera et al. (2016) and Tarr et al. (2016), the seven articles in a special issue on advances in data mining and robust

statistics as well as articles in regular issues such as Atkinson et al. (2016), Boente and Pardo-Fernández (2016), Chee and

Wang (2016), Cheng (2016), García-Escudero et al. (2016), Gutiérrez et al. (2016), Huang et al. (2016), Leung et al. (2016),

Li et al. (2016), Mount et al. (2016), Xiang et al. (2016), Aeberhard et al. (2017), Agostinelli et al. (2017), Bianco and Spano

(2017), Kaffine and Davis (2017), Maronna and Yohai (2017), Moradi Rekabdarkolaee et al. (2016), Serfling and Wijesuriya 
(2017) and Song et al. (2017), to just focus on publications since 2016.

We encourage all readers to continue to choose CSDA as one of their main outlets to share their latest achievements in the various important areas of robust statistics.

\title{
References
}

Aeberhard, W., Cantoni, E., Heritier, S., 2017. Saddlepoint tests for accurate and robust inference on overdispersed count data. Comput. Statist. Data Anal. 107, 162-175.

Agostinelli, C., Locatelli, I., Marazzi, A., Yohai, V., 2017. Robust estimators of accelerated failure time regression with generalized log-gamma errors. Comput. Statist. Data Anal. 107, 92-106.

Alfons, A., Croux, C., Gelper, S., 2016. Robust groupwise least angle regression. Comput. Statist. Data Anal. 93, 421-435.

Atkinson, A., Riani, M., Torti, F., 2016. Robust methods for heteroskedastic regression. Comput. Statist. Data Anal. 104, $209-222$.

Bali, J., Boente, G., 2017. Robust estimators under a functional common principal components model. Comput. Statist. Data Anal. x, 1-17.

Bianco, A. Spano, P., 2017. Robust estimation in partially linear errors-in-variables models. Comput. Statist. Data Anal. 106, 46-64.

Boente, G., Pardo-Fernández, J., 2016. Robust testing for superiority between two regression curves. Comput. Statist. Data Anal. 97, 151-168.

Candes, E., Tao, T., 2007. The dantzig selector: statistical estimation when p is much larger than n. Ann. Statist. 35, 2313-2351.

Chee, C., Wang, Y., 2016. Nonparametric estimation of species richness using discrete k-monotone distributions. Comput. Statist. Data Anal. 93, 107-118.

Cheng, C., 2016. Exploratory failure time analysis in large scale genomics. Comput. Statist. Data Anal. 95, 192-206.

Chiancone, A., Forbes, F., Girard, S., 2017. Student sliced inverse regression. Comput. Statist. Data Anal. x, 1-16.

Cook, R., 2007. Fisher lecture: Dimension reduction in regression. Statist. Sci. 22, 1-26.

Croux, C. Ronchetti, E., Salibián-Barrera, M., Van Aelst, S., 2013. Special issue on robust analysis of complex data. Comput. Statist. Data Anal. 65, 1-3.

Cui, Y., Leng, C Sun, D. 2016 Sparse estimation of high-dimensional correlation matrices. Comput Statist. Data Anal 93, 390-403.

Dhaene, G., Zhu, Y., 2017. Median-based estimation of dynamic panel models with fixed effects. Comput. Statist. Data Anal. x, 1-26.

García-Escudero, L. Gordaliza, A., Greselin, F., Ingrassia, S., Mayo-Iscar, A., 2016. The joint role of trimming and constraints in robust estimation for mixtures of gaussian factor analyzers. Comput. Statist. Data Anal. 99, 131-147.

Gutiérrez, L., Mena, R., Ruggiero, M., 2016. A time dependent bayesian nonparametric model for air quality analysis. Comput. Statist. Data Anal. 95, 161175.

Hämäläinen, W., 2016. New upper bounds for tight and fast approximation of fishers exact test in dependency rule mining. Comput. Statist. Data Anal. 93, 469-482.

He, Y., Zhang, X., Wang, P., Zhang, L., 2017. High dimensional gaussian copula graphical model with fdr control. Comput. Statist. Data Anal. x, 1-18. Huang, L., Zhao, J., Wang, H., Wang, S., 2016. Robust shrinkage estimation and selection for functional multiple linear model through lad loss. Comput. Statist. Data Anal. 103, 384-400.

Kaffine, D., Davis, G., 2017. A multi-row deletion diagnostic for influential observations in small-sample regressions. Comput. Statist. Data Anal. 108, 133145 .

Kirschstein, T., Liebscher, S., Porzio, G., Ragozini, G., 2016. Minimum volume peeling: A robust nonparametric estimator of the multivariate mode. Comput. Statist. Data Anal. 93, 456-468.

Leung, A., Zhang, H., Zamar, R., 2016. Robust regression estimation and inference in the presence of cellwise and casewise contamination. Comput. Statist. Data Anal. $99,1-11$

Li, Q., Lian, H., Zhu, F., 2016. Robust closed-form estimators for the integer-valued garch $(1,1)$ model. Comput. Statist. Data Anal. 101, 209-225.

Maronna, R., Yohai, V., 2017. Robust and efficient estimation of multivariate scatter and location. Comput. Statist. Data Anal. 109, 64-75.

Martinez, W., Gray, J., 2016. Noise peeling methods to improve boosting algorithms. Comput. Statist. Data Anal. 93, 483-497.

Maruotti, A., Punzo, A., 2017. Model-based time-varying clustering of multivariate longitudinal data with covariates and outliers. Comput. Statist. Data Anal. $x, 1-22$

Moradi Rekabdarkolaee, H., Boone, E., Wang, Q., 2016. Robust estimation and variable selection in sufficient dimension reduction. Comput. Statist. Data Anal. 108, 146-157.

Mount, D., Netanyahu, N., Piatko, C., Wu, A., Silverman, R., 2016. A practical approximation algorithm for the Its estimator. Comput. Statist. Data Anal. 99, $148-170$.

Salibián-Barrera, S., Van Aelst, S., Yohai, V., 2016. Robust tests for linear regression models based on image-estimates. Comput. Statist. Data Anal. 93, $436-$

455

Serfling, R., Wijesuriya, U., 2017. Depth-based nonparametric description of functional data, with emphasis on use of spatial depth. Comput. Statist. Data Serling, R, Wist

Song, J., Oh, D., Kang, J., 2017. Robust estimation in stochastic frontier models. Comput. Statist. Data Anal. 105, $243-267$.

Tarr, G., Müller, S., Weber, N., 2016. Robust estimation of precision matrices under cellwise contamination. Comput. Statist. Data Anal. 93, 404-420.

Xiang, S., Yao, W., Seo, B., 2016. Semiparametric mixture: Continuous scale mixture approach. Comput. Statist. Data Anal. 103, 413-425.

\author{
Samuel Mueller* \\ University of Sydney, Australia \\ E-mail address: samuel.mueller@sydney.edu.au. \\ Graciela Boente \\ Universidad Buenos Aires, Argentina \\ E-mail address: gboente@dm.uba.ar. \\ Christophe Croux \\ KU Leuven, Belgium \\ E-mail address: christophe.croux@econ.kuleuven.be. \\ Juan Romo \\ Universidad Carlos III de Madrid, Spain \\ E-mail address: romo@est-econ.uc3m.es. \\ Stefan Van Aelst \\ Ghent University, Belgium \\ E-mail address: stefan.vanaelst@ugent.be.
}

\section{*Corresponding editor.}

